\title{
Semiclassical theory of proton transport in ice
}

\section{Author: Dong-Yun Kim \& V. Hugo Schmidt}

This is a postprint of an article that originally appeared in Canadian Journal of Physics in April 1967.

Kim, Dong-Yun, and V. Hugo Schmidt. "Semiclassical theory of proton transport in ice" Canadian Journal of Physics 45, no. 4 (April 1967): 1507-1516.

http://dx.doi.org/10.1139/p67-115

Made available through Montana State University's ScholarWorks

scholarworks. montana.edu 


\title{
SEMICLASSICAL THEORY OF PROTON TRANSPORT IN ICE ${ }^{1}$
}

\author{
Dong-Yun KIm ${ }^{2}$ And V. Hugo Schmid' \\ Department of Physics, Montana State University, Bozeman, Montana
}

Received October 5, 1966

\begin{abstract}
A method is described for calculating proton or other ion mobility which is applicable if mobility is limited by lattice scattering rather than by barrier jumping. The Boltzmann transport equation is used, with the collision term calculated from the electrostatic interactions between the mobile ion and the vibrating lattice. In particular the proton mobility in ice is calculated. The lattice vibrations are approximated by a Debye spectrum for translational vibrations of water molecules, plus an Einstein spectrum for modes in which protons vibrate almost as independent particles. Scattering by phonons somewhat below the Debye cutoff frequency is of the greatest importance in determining the mobility, and the proton modes have negligible effect. The calculated mobility agrees reasonably well with the experimental value.
\end{abstract}

\section{INTRODUCTION}

Charge carrier mobilities in solids are governed either by lattice scattering or by thermally activated processes which transfer the carrier from one site to the next. For heavier ions and their corresponding vacancies in ionic crystals it has been generally found (Jost 1960) that mobility is governed by activation energies of the order of $1 \mathrm{eV}$. In ice, the activation energy for conductivity is due to creation of $\mathrm{H}_{3} \mathrm{O}^{+}-\mathrm{OH}^{-}$pairs, with effective motion of $\mathrm{H}_{3} \mathrm{O}^{+}$groups accomplished by means of protons moving along hydrogen bonds with no significant activation energy required (Eigen and De Maeyer 1958; Jaccard 1959; Onsager and Dupuis 1960, 1962; Riehl 1966). Accordingly, lattice scattering rather than hopping processes govern proton mobility in ice, and probably also in other hydrogen-bonded crystals which exhibit protonic conductivity of similar magnitude to that in ice.

The scattering cannot be calculated by the perturbational techniques commonly used for scattering of electrons because of the strong coupling between proton and lattice motions (Kittel 1963). Gosar and Pintar (1963, 1964), however, have made quantum mechanical calculations of proton mobility in ice. Owing to the invalidity of the usual perturbational method for the interaction of the proton-phonon system, they used the WKB method in order to treat the second-order process. Because of the nature of the approximation used, the validity of the Gosar-Pintar method is limited to the hopping process. However, the transport of protons in ice is governed by lattice scattering rather than by hopping processes (Eigen and De Maeyer 1958; Jaccard 1959; Onsager and Dupuis 1960, 1962; Rieh1 1966). Therefore, it is necessary to use a method which describes the proton transport in terms of lattice scattering and is independent of the perturbation expansion. Consequently, we have treated this subject by semiclassical approach.

${ }^{1}$ This work supported by National Institutes of Health Grant GM 12383 and by National Aeronautics and Space Administration Grant NsG-430.

${ }^{2}$ Present address: Department of Physics, University of Saskatchewan, Regina, Saskatchewan. 
We treat the mobile proton as a point particle obeying the laws of classical mechanics, except that the quantized nature of energy transfer between proton and lattice is taken into account. The point particle approximation is reasonably valid as the de Broglie wavelength of a thermal proton is less than $2 \AA$. Because the proton is in thermal equilibrium with the lattice, its energy gain from the lattice equals its loss to the lattice except for a slight energy gain from the applied electric field. In finding the energy loss to the lattice in terms of the transition probability for the creation of phonons, lattice thermal motion is ignored, as in the usual classical energy-loss calculation. Detailed balancing is then used to relate these probabilities to those for absorption of phonons from the lattice. These transition probabilities are used in the Boltzmann transport equation to calculate the mobility.

\section{GENERAL FORMALISM FOR MOBILITY}

For equilibrium, the velocity distribution function $f(\boldsymbol{u})$ for the mobile protons must satisfy the Boltzmann transport equation:

$$
\frac{\partial f(\boldsymbol{u})}{\partial t}=-\frac{\mathrm{d} \boldsymbol{u}}{\mathrm{d} t} \nabla \boldsymbol{u} f(\boldsymbol{u})-f(\boldsymbol{u}) \int w\left(\boldsymbol{u}, \boldsymbol{u}^{\prime}\right) \mathrm{d} \boldsymbol{u}^{\prime}+\int f\left(\boldsymbol{u}^{\prime}\right) w\left(\boldsymbol{u}^{\prime}, \boldsymbol{u}\right) \mathrm{d} \boldsymbol{u}^{\prime}=0 .
$$

The first and second integrals respectively describe scattering out of, and into, an element of velocity space at $\boldsymbol{u}$. We define a transition probability

$$
\text { (2) } w(u) \equiv \int w\left(\boldsymbol{u}, \boldsymbol{u}^{\prime}\right) \mathrm{d} \boldsymbol{u}^{\prime} \equiv \int_{0}^{m u^{2} / 2 \hbar} w\left(u, \omega_{0}\right) \mathrm{d} \omega_{0}+\int_{0}^{\infty} w\left(u,-\omega_{0}\right) \mathrm{d} \omega_{0},
$$

and assume that $w(u)$ is isotropic and field-independent. Here $\omega_{0}$ is positive for phonons emitted by the mobile proton, and the condition $\hbar \omega_{0}<\frac{1}{2} m u^{2}$ determines the upper limit for the first integral. We separate $f(\boldsymbol{u})$ into an isotropic term $f_{0}(u)$ independent of the applied field $\epsilon$ and a much smaller term $\delta f(\boldsymbol{u}, \boldsymbol{\epsilon})$. Then eq. (1) becomes

$$
\begin{aligned}
-\frac{e \epsilon}{m} \cos \theta \frac{\mathrm{d} f_{0}(u)}{\mathrm{d} u} & -\frac{e \boldsymbol{\varepsilon}}{m} \nabla_{\boldsymbol{u}} \delta f(\boldsymbol{u}, \boldsymbol{\varepsilon})-f_{0}(u) w(u)-\delta f(\boldsymbol{u}, \boldsymbol{\varepsilon}) w(u) \\
& +\int f_{0}\left(u^{\prime}\right) w\left(\boldsymbol{u}^{\prime}, \boldsymbol{u}\right) \mathrm{d} \boldsymbol{u}^{\prime}+\int \delta f\left(\boldsymbol{u}^{\prime}, \boldsymbol{\varepsilon}\right) w\left(\boldsymbol{u}^{\prime}, \boldsymbol{u}\right) \mathrm{d} \boldsymbol{u}^{\prime}=0,
\end{aligned}
$$

where $\theta$ is the angle between $\boldsymbol{u}$ and $\epsilon$. The second term is negligible, and the third and fifth terms cancel because these are the only terms in the zero-field Boltzmann equation. We substitute the solution

$$
\delta f(\boldsymbol{u}, \varepsilon)=-\frac{e \epsilon}{m} \frac{\cos \theta}{w(u)} \frac{\mathrm{d} f_{0}(u)}{\mathrm{d} u} G(u)
$$

into eq. (3) and in the last term replace $\cos \theta^{\prime}$ by $\cos \alpha \cos \theta+\sin \alpha \sin \theta \cos \phi$, where $\alpha$ is the angle between $\boldsymbol{u}$ and $\boldsymbol{u}^{\prime}$, and $\phi$ is the angle between the projections of $\boldsymbol{u}^{\prime}$ and $\boldsymbol{\varepsilon}$ on the plane perpendicular to $\boldsymbol{u}$. For an isotropic material, the integral containing $\cos \phi$ vanishes, and eq. (3) becomes

$$
G(u)-\left[\frac{\mathrm{d} f_{0}(u)}{\mathrm{d} u}\right]^{-1} \int \frac{\cos \alpha}{w\left(u \iota^{\prime}\right)} \frac{\mathrm{d} f_{0}\left(u^{\prime}\right)}{\mathrm{d} u^{\prime}} G\left(u^{\prime}\right) w\left(\boldsymbol{u}^{\prime}, \boldsymbol{u}\right) \mathrm{d} \boldsymbol{u}^{\prime}=1 .
$$


To simplify the following calculations we assume isotropic scattering, for which $w\left(\boldsymbol{u}, \boldsymbol{u}^{\prime}\right)$ is independent of $\alpha$, and this integral equation then reduces to $G(u)=$ 1 . This is a reasonably accurate assumption for scattering by water molecules because of their relatively great mass. For bound protons this assumption gives scattering roughly twice the correct value, but it will be shown that even with this overestimated value, scattering from bound protons has a negligible effect on the mobility.

The drift velocity is obtained by in tegrating $\boldsymbol{u} f(\boldsymbol{u})$ over velocity space. Only the $\delta f(\boldsymbol{u}, \boldsymbol{\varepsilon})$ term gives a contribution, which by symmetry is in the field direction:

$$
\begin{aligned}
V_{\mathrm{d}} & =\int_{0}^{\infty} \int_{0}^{\pi} \boldsymbol{u} \delta f(\boldsymbol{u}, \boldsymbol{\varepsilon}) 2 \pi u^{2} \sin \theta \mathrm{d} \theta \mathrm{d} u \\
& =-\frac{2 \pi e \varepsilon}{m} \int_{0}^{\infty} \int_{0}^{\pi} \frac{\sin \theta \cos ^{2} \theta \mathrm{d} \theta u^{3} \mathrm{~d} f_{0}(u) / \mathrm{d} u}{w(u)} \mathrm{d} u .
\end{aligned}
$$

The mobility $\mu=V_{\mathrm{d}} / \varepsilon$ is then given by

$$
\mu=-\frac{4}{3} \pi \frac{e}{m} \int_{0}^{\infty} \frac{u^{3}}{w(u)} \frac{\mathrm{d} f_{0}(u)}{\mathrm{d} u} \mathrm{~d} u,
$$

which is positive for positive carriers for the usual negative $\mathrm{d} f_{0}(u) / \mathrm{d} u$.

In calculating $w(u)$ from eq. (2), the expressions for $w\left(u, \omega_{0}\right)$ derived in the following sections hold only for positive $\omega_{0}$. To take into account negative $\omega_{0}$ (phonons absorbed from the lattice), we assume detailed balancing, which requires that the rate at which protons initially in an energy interval $\mathrm{d} E$ at $E \equiv \frac{1}{2} m u^{2}$ absorb quanta $\hbar \omega_{0}$ and enter the interval $\mathrm{d} E$ at $E+\hbar \omega_{0}$ must equal the rate at which they drop from this interval to the interval $\mathrm{d} E$ at $E$ by emitting quanta $\hbar \omega_{0}$. The interval $\mathrm{d} E$ has volume $4 \pi u m^{-1} \mathrm{~d} E$ in velocity space, so the probability that a given proton is in an element $\mathrm{d} E$ is $.4 \pi u m^{-1} f_{0}(u) \mathrm{d} E$, and detailed balancing requires that

$$
4 \pi u m^{-1} f_{0}(u) w\left(u,-\omega_{0}\right) \mathrm{d} E=4 \pi u^{\prime} m^{-1} f_{0}\left(u^{\prime}\right) w\left(u^{\prime}, \omega_{0}\right) \mathrm{d} E,
$$

where $\frac{1}{2} m u^{\prime 2}=\frac{1}{2} m u^{2}+\hbar \omega_{0}$. The above relation inserted into eq. (2) yields

$$
w(u)=\int_{0}^{m u^{2} / 2 \hbar} w\left(u, \omega_{0}\right) \mathrm{d} \omega_{0}+\int_{0}^{\infty} \frac{u^{\prime}}{u} \cdot \frac{f_{0}\left(u^{\prime}\right)}{f_{0}(u)} \cdot w\left(u^{\prime}, \omega_{0}\right) \mathrm{d} \omega_{0} .
$$

Substitution of eq. (9) into eq. (7) gives the following expression for the mobility:

$$
\begin{aligned}
\mu=-\frac{4}{3} \pi \frac{e}{m} & \int_{0}^{\infty} u \frac{\mathrm{d} f_{0}(u)}{\mathrm{d} u} \\
& \times\left[\int_{0}^{m u^{2} / 2 \hbar} w\left(u, \omega_{0}\right) \mathrm{d} \omega_{0}+\int_{0}^{\infty} \frac{u^{\prime} f_{0}\left(u^{\prime}\right)}{u f_{0}(u)} w\left(u^{\prime}, \omega_{0}\right) \mathrm{d} \omega_{0}\right]^{-1} \mathrm{~d} u .
\end{aligned}
$$

The assumptions about the proton-phonon interaction contained in the frame of the semiclassical approach are those of isotropic scattering, detailed balance, and lack of activation energy for the mobility. As is characteristic of the semiclassical method, only creation (or annihilation) of one phonon has 
been taken into account (normal process). According to this method, it is possible to give a correct and plausible explanation of the effect of a phonon field on a system of particles (creation and annihilation of phonons) but not of the influence of particles on the phonon field: in general, the "law of conservation of momentum" does not hold, i.e. one has to consider the Umklapp process, which can be interpreted as the creation (or annihilation) of phonons with additional momentum (Bragg reflection) which is transferred to the "crystal reservoir" as a whole.

The interactions of protons with the lattice defects and impurities are negligible compared with the proton-lattice interaction, because the mean free path of the mobile proton turns out to be only about $1 \AA$. The average separation of defects or impurities is much greater than $1 \AA$. For instance, the concentration at $-10^{\circ} \mathrm{C}$ of mobile protons and of vacant hydrogen bonds is approximately $10^{11} / \mathrm{cm}^{3}$ (Onsager and Dupuis 1962), so their mean separation is of the order of $10^{4} \AA$.

Scattering of mobile protons both by proton vibrational modes and by water molecule vibration modes is considered. The bound proton, because of its small mass, can be approximated by a single-particle harmonic oscillator. For the lower frequency modes in which the water molecules move in translation approximately as rigid units, the actual phonon spectrum is approximated by a Debye spectrum. We assume that the interaction of the mobile proton with the $3 N$ Debye modes is equivalent to its interaction with $N$ single-particle oscillators having the water molecule mass and electric dipole moment and having spectral density $g\left(\omega_{0}\right)$ proportional to $\omega_{0}{ }^{2}$, corresponding to the Debye spectrum. This assumption neglects correlations between forces exerted by the mobile proton on a given water molecule dipole and forces exerted on its neighbors. Such correlations are small because the water molecule orientation in ice is relatively random.

The carrier motion in ice consists of effective motion of four types of defects (Eigen and De Maeyer 1958; Jaccard 1959; Onsager and Dupuis 1960, 1962; Riehl 1966). Transfer of $\mathrm{H}_{3} \mathrm{O}^{+}$and $\mathrm{OH}^{-}$ions occurs by means of proton motions along successive hydrogen bonds. Bjerrum $L$ and $D$ defects are propagated by rotations of successive water molecules resulting in proton transfer from one bond to another. In each case the mobile proton serving as current carrier changes identity each time the defect moves a lattice space. Our study of a single mobile proton in place of this series of carriers is based on the assumption that lattice scattering destroys correlations between motions of these successive carriers in the same manner as it destroys the correlations in time of the motion of our single carrier. We approximate the crystal by a simple cubic array of oscillators, with the mobile proton moving along one line of oscillators at any given instant. The minimum impact parameter $b_{0}$ is then (oscillator density) $)^{-\frac{1}{3}}$. Our calculational method is not suitable for consideration of scattering by molecules along the path of the mobile proton. Such scattering is probably at least as important as scattering by all the other oscillators.

The transition probability $w\left(u, \omega_{0}\right)$ for transfer of a quantum of energy $\hbar \omega_{0}$ from the mobile proton to oscillators of that frequency is found by dividing the 
classical rate of energy loss to oscillators of that frequency by the quantum energy (Jackson 1962):

$$
w\left(u, \omega_{0}\right)=\frac{\mathrm{d} E\left(u, \omega_{0}\right)}{\mathrm{d} t}\left(\hbar \omega_{0}\right)^{-1} .
$$

The energy loss $\delta E\left(u, \omega_{0}, b\right)$ to an individual oscillator is calculated for a mobile proton of mass $m$ going past an oscillator of mass $M$ and natural frequency $\omega_{0}$ at constant velocity $\boldsymbol{u}$ with impact parameter $b$. The energy-loss rate per unit frequency interval is found by integrating the product of energy loss and oscillator density over $b$, and multiplying by the mobile proton velocity:

$$
\frac{\mathrm{d} E\left(u, \omega_{0}\right)}{\mathrm{d} t}=u g\left(\omega_{0}\right) \int_{b_{0}}^{\infty} \delta E\left(u, \omega_{0}, b\right) 2 \pi b \mathrm{~d} b .
$$

The approximation that oscillator distribution is continuous and uniform for $b>b_{0}$ is used in this integration, which, together with the integration over $u$ in eq. (10), approximates fairly well an integration over the actual proton paths, which consist of short segments, each having its own value of $b$ and $\boldsymbol{u}$ because of the low (thermal) proton kinetic energies.

The calculation of energy loss for a charged particle moving uniformly past an oscillator initially at rest is well known (Jackson 1962) and is only briefly outlined here. The equation of motion of an isotropically bound particle of natural frequency $\omega_{0}$ and damping factor $\Gamma$ under the influence of a force $\boldsymbol{F}(t)$ due to the moving charge is

$$
\ddot{\boldsymbol{X}}+\Gamma \dot{X}+\omega_{0}^{2} \boldsymbol{X}=\boldsymbol{F}(t) / M
$$

In terms of Fourier components of force and position, the solution is

$$
X(\omega)=\frac{F(\omega) / M}{\omega_{0}^{2}-\omega^{2}-i \Gamma \omega} .
$$

In the approximation that $\Gamma$ is negligible, the energy transfer to the initially stationary oscillator is given by

$$
\delta E\left(u, \omega_{0}, b\right)=(\pi / M)\left|\boldsymbol{F}\left(\omega_{0}\right)\right|^{2} .
$$

The electrostatic force on the bound particle at its actual position is assumed to be the same as at its equilibrium position. The oscillating protons are treated as partly shielded point charges, while the oscillating water molecules are approximated by point dipoles, so separate calculations of the force must be made for these two cases.

\section{SCATTERING BY PROTON VIBRATIONAL MODES}

The mobile proton exerts an electrostatic force $z e^{2} / r^{2}$ on the bound proton, where $z$ is a shielding factor. The energy transfer for this force has been found (Jackson 1962) to be

$$
\delta E=\frac{2 z^{2} e^{4}}{M u^{2} b^{2}}\left[\eta^{2} K_{1}^{2}(\eta)+\eta^{2} K_{0}^{2}(\eta)\right],
$$


where $\eta=b \omega_{0} / u$ and $K_{0}$ and $K_{1}$ are modified Bessel functions. For $\eta \ll 1$, the square bracket in eq. (16) approaches unity. The only case of importance for energy loss to proton vibrational modes will be shown to be $\eta \gg 1$, for which the square bracket becomes $\pi \eta \mathrm{e}^{-2 \eta}$, giving an energy transfer of

$$
\delta E=\frac{2 \pi z^{2} e^{4} \eta \mathrm{e}^{-2 \eta}}{M u^{2} b^{2}} .
$$

Substitution of this energy transfer into eqs. (11) and (12) yields

$$
w\left(u, \omega_{0}\right)=\frac{2 \pi^{2} z^{2} e^{4} g\left(\omega_{0}\right)}{M u \hbar \omega_{0}} \exp \left(-\frac{2 b_{0} \omega_{0}}{u}\right) .
$$

If oscillators of only one frequency $\omega_{0}$, having density $N$ per unit volume, are important in determining the mobility, and if a Maxwellian distribution

$$
f_{0}(\boldsymbol{u})=\left(\frac{m}{2 \pi k T}\right)^{3 / 2} \exp \left(-\frac{m u^{2}}{2 k T}\right)
$$

is assumed for the mobile protons, eq. (9) becomes

$$
w(u)=\frac{2 \pi^{2} z^{2} e^{4} N}{M u \hbar \omega_{0}} \cdot\left\{\exp \left(-2 b_{0} \omega_{0} / u\right)+\left(u^{\prime} / u\right) \exp \left(-\frac{\hbar \omega_{0}}{k T}-\frac{2 b_{0} \omega_{0}}{u^{\prime}}\right)\right\} .
$$

For $\hbar \omega_{0} \equiv \frac{1}{2} m u_{0}^{2}>\frac{1}{2} m u^{2}$, the term $\exp \left(-2 b_{0} \omega_{0} / u\right)$ must be dropped. The above expression substituted into eq. (7) for the mobility gives

$$
\begin{aligned}
\mu=\frac{4}{3} & \frac{M}{m}\left(\frac{m}{2 \pi k T}\right)^{5 / 2} \frac{\hbar \omega_{0}}{z^{2} e^{3} N} \cdot\left\{\int_{0}^{u u_{0}} \frac{u^{6}}{u^{\prime}} \exp \left(\frac{\hbar \omega_{0}-\frac{1}{2} m u^{2}}{k T}+\frac{2 b_{0} \omega_{0}}{u^{\prime}}\right) \mathrm{d} u\right. \\
& \left.+\int_{0}^{\infty} \frac{u^{5} \exp \left(-m u^{2} / 2 k T\right)}{\exp \left(-2 b_{0} \omega_{0} / u\right)+\left(u^{\prime} / u\right) \exp \left(-\hbar \omega_{0} / k T-2 b_{0} \omega_{0} / u\right)} \mathrm{d} u\right\} .
\end{aligned}
$$

The first integral takes account of the slower protons which can only absorb quanta from the oscillators, while the second integral concerns protons which can either absorb or emit quanta of energy $\hbar \omega_{0}$.

Because $w(u)$ is the only frequency-dependent factor in eq. (7) for the mobility, and $w(u)$ decreases rapidly with increasing frequency in the range of the proton modes, only the lowest frequency mode is considered. This mode has been found (Giguere and Harvey 1956) by infrared absorption to be the $\mathrm{H}_{2} \mathrm{O}$ libration (restricted rotation) mode near $800 \mathrm{~cm}^{-1}$, for which $\omega_{0}$ is $1.51 \times$ $10^{14} / \mathrm{sec}$. Dynamically the motion is almost equivalent to vibration of two rigidly coupled protons, and accordingly the oscillator mass $M$ is taken as $2 m$ and the oscillator density is the $\mathrm{H}_{2} \mathrm{O}$ group density $N$. The minimum impact parameter $b_{0}$ is $(2 N)^{-\frac{1}{3}}$, or $2.54 \AA$.

The effective charge $z e$ of the bound proton is assumed proportional to its degree of ionic character, so that $z$ is taken as 0.34 , the value given by Pauling (1960) for the relative contribution of the ionic structure to the hydrogen bond in ice. In MKS units $z$ must be divided by $4 \pi \epsilon_{0}$. No additional shielding of the bound protons is assumed, because the factor $\exp \left(-2 b \omega_{0} / \imath\right)$ in eq. (17) causes 
the scattering to be due chiefly to the very nearest bound protons, for which the bulk dielectric constant does not apply.

In evaluating the mobility at $-10^{\circ} \mathrm{C}$ from eq. (21) using the above parameters, only the first integral gives a significant contribution. This integral is for velocities from zero to $u_{0}=\left(2 \hbar \omega_{0} / m\right)^{\frac{1}{2}}=4.37 \times 10^{3} \mathrm{~m} / \mathrm{sec}$. Because $b_{0} \omega_{0}=$ $3.84 \times 10^{4} \mathrm{~m} / \mathrm{sec}$, the approximation $b \omega_{0} / u \gg 1$ made in deriving eq. (17) is found to be valid.

The proton mobility in ice, calculated on the basis of scattering by the 800 $\mathrm{cm}^{-1}$ proton mode only, is $4.0 \times 10^{3} \mathrm{~cm}^{2} /$ volt-second at $-10^{\circ} \mathrm{C}$. A modified calculation, which allows mobile protons to approach bound protons somewhat closer than $b_{0}$ by an amount depending on their kinetic energy, yields a mobility of $2.4 \times 10^{2} \mathrm{~cm}^{2} /$ volt-second. This illustrates the strong dependence on $b_{0}$ of scattering by modes in this frequency range; but even with this modification, the calculated mobility is about three orders of magnitude greater than the experimental value (Eigen and De Maeyer 1958; Eigen, De Maeyer, and Spatz 1962). This high mobility, corresponding to small transition probability $w(u)$ for quantum exchange between mobile proton and oscillator, occurs because the bound proton pair, idealized as a single particle in a potential well, can respond almost adiabatically to the field of a mobile proton having thermal velocity. Also the quantum energy for the $800 \mathrm{~cm}^{-1}$ mode is greater than $4 k T$ and few of the mobile protons or oscillators have this much energy to give up. Accordingly, lower frequency modes associated with $\mathrm{H}_{2} \mathrm{O}$ translational modes, for which the single particle oscillator approximation cannot be used, give a larger transition probability and correspondingly lower mobility.

\section{EFFECT OF $\mathrm{H}_{2} \mathrm{O}$ VIBRATIONAL MODES ON MOBILITY}

The water molecules are treated as permanent electric dipoles of moment $\boldsymbol{u}$ having random orientation relative to the mobile proton path. The electrostatic force exerted by the proton on the dipoles is reduced by the dielectric constant $\epsilon$ appropriate to the frequency components of the electric field due to the moving proton. We define $\mu_{1}=\mu \cos \beta$ as the component of dipole moment parallel to the proton velocity $\boldsymbol{u}$. The transverse component which experiences a net force is $\mu_{\mathrm{t}}=\mu \sin \beta \cos \gamma$, where $\gamma$ is the angle in the plane perpendicular to $\boldsymbol{u}$ between the vector from the dipole to the proton path and the projection of the dipole moment vector onto this plane. The third component of dipole moment, $\mu \sin \beta \sin \gamma$, feels no net force, but experiences a torque which gives rise to a high frequency rotational mode that has little effect on the mobility. Taking $t=0$ at the moment of closest approach, the force on the dipole has a component parallel to $\boldsymbol{u}$ containing two terms:

$$
F_{1}=F_{11}+F_{1 \mathrm{t}}=-\left(2 e / \epsilon r^{5}\right)\left(\mu_{1} u^{2} t^{2}+\mu_{\mathrm{t}} b u t\right), \quad r^{2}=b^{2}+u^{2} t^{2} .
$$

The other force component, perpendicular to $\boldsymbol{u}$ and directed towards the proton path, has the two terms

$$
F_{\mathrm{t}}=F_{\mathrm{t} 1}+F_{\mathrm{tt}}=-\left(2 e / \epsilon r^{5}\right)\left(\mu_{1} b u t+\mu_{\mathrm{t}} b^{2}\right) .
$$

The Fourier transforms of these forces are given by 


$$
\begin{aligned}
& F_{11}(\omega)=-\frac{2}{3} \sqrt{\frac{2}{\pi}} \mu_{1} \frac{e}{\epsilon} \frac{\omega^{2}}{u^{3}}\left[\frac{u}{b \omega} K_{1}\left(\frac{b \omega}{u}\right)-K_{0}\left(\frac{b \omega}{u}\right)\right], \\
& F_{1 \mathrm{t}}(\omega)=-\frac{2}{3} i \sqrt{\frac{2}{\pi}} \mu_{\mathrm{t}} \frac{e}{\epsilon} \frac{\omega^{2}}{u^{3}} K_{1}\left(\frac{b \omega}{u}\right), \\
& F_{\mathrm{t} 1}(\omega)=-\frac{2}{3} i \sqrt{\frac{2}{\pi}} \mu_{1} \frac{e}{\epsilon} \frac{\omega^{2}}{u^{3}} K_{1}\left(\frac{b \omega}{u}\right), \\
& F_{\mathrm{tt}}(\omega)=-\frac{2}{3} \sqrt{\frac{2}{\pi}} \mu_{\mathrm{t}} \frac{e}{\epsilon} \frac{\omega^{2}}{u^{3}}\left[\frac{2 u}{b \omega} K_{1}\left(\frac{b \omega}{u}\right)+K_{0}\left(\frac{b \omega}{u}\right)\right] .
\end{aligned}
$$

Here $K_{0}$ and $K_{1}$ again are modified Bessel functions. Squaring the longitudinal and transverse forces separately and substituting the sum of the squares into eq. (15) yields the following expression for energy loss:

$$
\begin{aligned}
\delta E\left(u, \omega_{0}, b\right)=\frac{8}{9} \frac{e^{2} \omega_{0}{ }^{4}}{M u^{6} \epsilon^{2}}\left\{\mu _ { t } { } ^ { 2 } \left[\frac{4 K_{1}{ }^{2}}{y^{2}}\right.\right. & \left.+\frac{4 K_{1} K_{0}}{y}+K_{0}{ }^{2}+K_{1}{ }^{2}\right] \\
& \left.+\mu_{1}{ }^{2}\left[\frac{K_{1}{ }^{2}}{y^{2}}-\frac{2 K_{1} K_{0}}{y}+K_{0}{ }^{2}+K_{1}{ }^{2}\right]\right\},
\end{aligned}
$$

where $y=b \omega_{0} / u$.

Taking an average of $\mu_{1}^{2}$ and $\mu_{t}^{2}$ over the solid angle yields the mean energy loss of the mobile proton per dipole for random dipole orientation:

$$
\overline{\delta E\left(u, \omega_{0}, b\right)}=\frac{16 \boldsymbol{u}^{2} e^{2} \omega_{0}^{4}}{27 \epsilon^{2} M u^{6}}\left[\frac{5 K_{1}^{2}(y)}{2 y^{2}}+\frac{K_{1}(y) K_{0}(y)}{y}+K_{1}{ }^{2}(y)+K_{0}{ }^{2}(y)\right] .
$$

Substitution of this expression into eq. (12) and changing the variable of integration from $b$ to $y$ gives for the transition probability per unit frequency in eq. (11) the expression

$$
w\left(u, \omega_{0}\right)=\frac{32 \pi \mu^{2} e^{2} \omega_{0} g\left(\omega_{0}\right)}{27 \epsilon^{2} M u^{3} \hbar} \int_{v_{0}}^{\infty} y\left[\frac{5 K_{1}{ }^{2}}{2 y^{2}}+\frac{K_{1} K_{0}}{y}+K_{1}{ }^{2}+K_{0}{ }^{2}\right] \mathrm{d} y .
$$

If the $N$ water molecule oscillators per unit volume are distributed according to a Debye spectrum with cutoff frequency $\omega_{\mathrm{c}}$, the oscillator density is

$$
g\left(\omega_{0}\right)=3 N \omega_{0}^{2} / \omega_{c}^{3} .
$$

The transition probability per unit frequency range then becomes

$$
w\left(u, \omega_{0}\right)=\frac{32 \pi N u^{2} e^{2} \omega_{0}^{3} F\left(y_{0}\right)}{9 \epsilon^{2} M \hbar \omega_{\mathrm{c}}{ }^{3} u^{3}},
$$

where $F\left(y_{0}\right)$ is the integral in eq. (27). The transition probability of eq. (9) can then be written as

$$
\begin{aligned}
w(u)=\frac{32 \pi N \boldsymbol{u}^{2} e^{2}}{9 \epsilon^{2} M \hbar \omega_{\mathrm{c}}{ }^{3} \bar{b}_{0}{ }^{4}} u\left\{\int_{0}^{\left(m u b_{0}\right) / 2 \hbar}\right. & y_{0}^{3} F\left(y_{0}\right) \mathrm{d} y_{0} \\
& \left.+\int_{0}^{\infty} y_{0}{ }^{3} \frac{F\left(y_{0}^{\prime}\right) \exp \left(-\hbar \imath y_{0} / k T b_{0}\right)}{1+\left(2 \hbar y_{0} / m u b_{0}\right)} \mathrm{d} y_{0}\right\} .
\end{aligned}
$$


The integrations over $y_{0}$ are in effect integrations over $\omega_{0}$. The upper limit for the first integral can be taken as infinity without appreciable error for the values of $u$ near $\overline{\boldsymbol{u}}=(8 k T / \pi m)^{\frac{1}{2}}=2.35 \times 10^{3} \mathrm{~m} / \mathrm{sec}$ at $-10^{\circ} \mathrm{C}$, which give the only significant contributions to the integral over $u$ in eq. (10) for the mobility. The value of this integral is found by numerical integration to be 1.24 . Evaluation of the second integral for $u \equiv \bar{u}$ gives a value of 1.22 , so that no important error results from assuming a value of 1.24 for the second integral also for all values of $u$. In this approximation, the mean free path $\lambda$ is independent of $u$ in the relation $v(u)=u / \lambda$, and is given by

$$
\lambda=0.0361 \frac{\epsilon^{2} M \hbar \omega_{c}{ }^{3} b_{0}{ }^{4}}{N u^{2} e^{2}} \text {. }
$$

For $\epsilon$ and $\boldsymbol{u}$ we use the values suggested by Onsager and Dupuis $(1960,1962)$ of 3.1 and $(0.765 \AA) e$ respectively. In MKS units, $\epsilon$ must be multiplied by $4 \pi \epsilon_{0}$. For $\omega_{\mathrm{c}}$ we use $4.50 \times 10^{13} / \mathrm{sec}$ corresponding to a wave number of $239 \mathrm{~cm}^{-1}$, which is slightly above the upper of the two infrared and Raman peaks listed (Pimentel and McClellan 1960) for ice at 160 and $212 \mathrm{~cm}^{-1}$ and attributed to translational motion of the water molecules. This cutoff frequency corresponds to the Debye temperature $\theta\left(263{ }^{\circ} \mathrm{K}\right)=344^{\circ} \mathrm{K}$ found by Flubacher, Leadbetter, and Morrison (1960). The molecular density $N$ is $3.06 \times 10^{22} / \mathrm{cm}^{3}$, so the minimum impact parameter $b_{0}$ taken as $N^{-\frac{1}{3}}$ is $3.20 \AA$. The symbol $M$ represents the mass of the water molecule. For these values of the parameters, the mean free path $\lambda$ is $1.10 \AA$.

Insertion of the approximate expression $w(u)=u / \lambda$ into eq. (7) and assumption of the Maxwellian distribution in eq. (21) for $f_{0}(u)$ yields the following expression for the mobility:

$$
\mu=e \lambda\left(\frac{8}{9 \pi m k T}\right)^{\frac{1}{2}}=\frac{8 e \lambda}{3 \pi m u} .
$$

This expression predicts a mobility at $-10^{\circ} \mathrm{C}$ of $3.8 \times 10^{-2} \mathrm{~cm}^{2} /$ volt-sec, somewhat under the experimental value (Eigen, De Maeyer, and Spatz 1962) $7.5 \times 10^{-2} \mathrm{~cm}^{2} /$ volt-sec. The agreement is quite good in view of the approximations made, and the uncertainties in the values of the various parameters, especially $b_{0}$. The present calculated value is somewhat above the value calculated by Gosar-Pinter $\left(2.5 \times 10^{-2} \mathrm{~cm}^{2} /\right.$ volt-sec at $\left.-10^{\circ} \mathrm{C}\right)$. The greater discrepancy between the Gosar-Pinter result and the experimental result may have been caused by the fact that they used the hopping model.

The calculation could be refined to take the actual (hexagonal) structure of ice into account. An anisotropy in the mobility would result. Two additional refinements, which would tend to lower the mobility, are (i) use of an effective dielectric constant lower than the bulk value for interactions with the nearest water molecules, and (ii) consideration of scattering by water molecules along the path of the mobile proton. It is the nature of the scattering, which is not isotropic as assumed in deriving eq. (2), but is strongly peaked in the forward direction, to tend to increase the mobility. This forward peaking occurs because in eq. (30) the major contributions to the integrals are for $b_{0} \omega_{0} / u$ near unity, 
for which the phonon momentum $\hbar k=\hbar \omega_{0} / v_{\mathrm{s}} \cong \hbar u / b_{0} v_{\mathrm{s}}$ is only about $1 / 10$ of the momentum of a thermal proton. Here $v_{5}$ is $2.18 \times 10^{3} \mathrm{~m} / \mathrm{sec}$, the velocity of sound in ice (Flubacher, Leadbetter, and Morrison 1960). The frequency $\vec{u} / b_{0}$, near which the major part of the scattering occurs, is $39 \mathrm{~cm}^{-1}$. The scattering falls off rapidly with increasing frequency, so the translational vibration peaks found (Pimentel and McClellan 1960) at 160 and $212 \mathrm{~cm}^{-1}$ near the Debye cutoff have little effect on the mobility.

The expression for mobility in eq. (32) includes no quantum effects, because in the first integral in eq. (30) the upper limit can be suppressed and in the second integral the quantum effects almost cancel. The absence of quantum effects occurs because the scattering is due primarily to phonons of energy much less than $k T$. The mobility is predicted to increase with decreasing temperature, as was found experimentally by Riehl (1966). Because deuteron mobility in heavy ice is reported (Riehl 1966) to require activation energy, a different material must be used to test the predicted mobility decrease with increasing carrier mass.

\section{ACKNOWLEDGMENTS}

We should like to thank J. A. Ball, K. L. Nordtvedt, D. K. Anderson, and others in the Physics Department at Montana State University for many stimulating discussions. We are indebted also to R. T. Shuey for helpful suggestions.

\section{REFERENCES}

Eigen, M. and De Maeyer, L. 1958. Proc. Roy. Soc. (London), Ser. A, 247, 505.

Eigen, M., De Maeyer, L., and Sratz, H. C. 1962. Coll. Physics of Ice Crystals (Erlenbach, Zurich)

Flubacher, P., Leadbetter, A. J., and Morrison, J. A. 1960. Chem. Phys. 33, 1751.

Giguere, P. A. and Harvey, K. B. 1956. Can. J. Chem. 34, 798.

Gosar, P. 1963. Nuovo cimento, 30, 931.

Gosar, P. and Pintar, M. 1964. Phys. Stat. Sol. 4, 675.

JACCARD, C. 1959 . Helv. Phys. Acta, 32, 89.

JACKson, J. D. 1962 . Classical electrodynamics, chap. 13 (Wiley, New York).

Jost, W. 1960. Diffusion, chap. IV (Academic Press, New York).

KitTEL, C. 1963. Quantum theory of solids (Wiley, New York), p. 134.

Onsager, L., and Dupuis, M. 1960. Rend. S.I.F., X Corso (Bologna), 294.

Paut 1962 . Electrolytes (Pergamon Press, New York), p. 27.

Pauling, L. 1960. The nature of the chemical bond, 3rd ed. (Cornell Univ. Press, Ithaca, N.Y.), pp. 78,453 .

Pimentel, G. C. and MCClellan, A. L. 1960. The hydrogen bond (W. H. Freeman and Co., San Francisco), p. 134

Riehl, N. 1966. Energy transfer in radiation processes (Elsevier, Amsterdam), p. 95. 